\title{
Mechanistic insights into the distinct functions of ATG16L1 in canonical autophagy and selective autophagy
}

\section{Lifeng Pan ( $\square$ panlf@sioc.ac.cn )}

Shanghai Institute of Organic Chemistry https://orcid.org/0000-0002-9229-6288

\section{Xingyu Gong}

Shanghai Institute of Organic Chemistry

\section{Yingli Wang}

Shanghai Institute of Organic Chemistry

\section{Yubin Tang}

Shanghai Institute of Organic Chemistry

Jianping Liu

Shanghai Institute of Organic Chemistry https://orcid.org/0000-0001-7090-2112

\section{Ying Li}

Shanghai Institute of Organic Chemistry

\section{Yaru Wang}

Shanghai Institute of Organic Chemistry

\section{Xiaolong Xu}

Shanghai Institute of Organic Chemistry

\section{Mingfang Zhang}

Shanghai Institute of Organic Chemistry

\section{Miao Li}

Shanghai Institute of Organic Chemistry

\section{Yuchao Zhang}

Shanghai Institute of Organic Chemistry

\section{Liqiang Shen}

Shanghai Institute of Organic Chemistry

\section{Article}

Keywords: ATG16L1, WIPI2b, RB1CC1, ATG8 family proteins, Autophagy receptor

Posted Date: July 12th, 2021

DOI: https://doi.org/10.21203/rs.3.rs-660487/v1 
License: (c) (i) This work is licensed under a Creative Commons Attribution 4.0 International License. Read Full License 


\section{Abstract}

Macroautophagy plays crucial roles in the regulation of cellular physiology, and requires de novo synthesis of double-membrane autophagosomes. The formation of autophagosomes entails the lipidation of ATG8 family proteins catalyzed by the ATG12 ATG5-ATG16L1 complex, which is recruited to the pre-autophagosomal membrane through a direct interaction between ATG16L1 and WIPI2b. In addition, ATG16L1 can function as an adaptor to specifically recognize invading pathogens to mediate antibacterial selective autophagy, and the T300A polymorphism of ATG16L1 has been associated with the Crohn's disease, an inflammatory bowel disease mainly caused by the defect in antibacterial autophagy. However, the molecular mechanism governing the interaction of ATG16L1 with WIPI2b and the precise role of ATG16L1 in selective autophagy remain elusive. Here, we reported the atomic structure of the WIPI2b/ATG16L1 complex, and elucidated the mechanistic basis underpinning the recruitment of ATG16L1 by WIPI2b. Moreover, we discovered that the FIR motif of ATG16L1 not only can directly interact with RB1CC1 Claw domain, but also can selectively recognize mammalian ATG8 family proteins. We determined the high-resolution crystal structures of ATG16L1 FIR in complex with RB1CC1 Claw and GABARAPL1 respectively, and uncovered the molecular mechanism underlying the interactions of ATG16L1 with RB1CC1 and ATG8 family proteins. On this basis, we proposed the concept that ATG16L1 can be categorized as a potential autophagy receptor. In all, our findings provide novel mechanistic insights into the interactions of ATG16L1 with WIPI2b, RB1CC1 and ATG8 family proteins, and are valuable for further understanding the distinct functions of ATG16L1 in autophagy.

\section{Introduction}

Macroautophagy (hereafter referred to as autophagy) is a well conserved and coordinated catabolic process involving lysosome-dependent degradation of undesired or harmful cytosolic components, such as bulk protein aggregates, dysfunctional organelles and invasive pathogens, for maintaining cellular homeostasis and physiology in mammals ${ }^{1-6}$. During autophagy, the encapsulation of autophagic cargoes for delivery to lysosomes relies on the unique double-membraned vesicles termed as autophagosomes, which are generated through several sequential steps mediated by the coordinated actions of a series of core autophagic proteins ${ }^{5-9}$. In particular, in non-selective canonical autophagy, such as the starvation-induced autophagy, the ULK complex, which is composed of ULK1/2 kinase, ATG13, ATG101 and RB1CC1, plays a critical role in phagophore initiation by recruiting and phosphorylating multiple downstream factors $5,6,8,9$. Then, the activated class III phosphatidylinositol 3kinase complex I (PI3KC3-C1) generates phosphatidylinositol 3-phosphate (PI3P) to act in phagophore nucleation ${ }^{5,6,8}$. Subsequently, the PI3P generated by PI3KC3-C1 can be specifically sensed by the downstream WD-repeat protein interacting with phosphoinositide (WIPI) proteins, which consist of four members in mammalian (WIPI1 to WIPI4) and can further recruit additional PI3KC3-C1 as well as other binding partners, such as ATG16L1 and ATG2A/2B, to promote phagophore elongation at the site of PI3P

generation $5,6,8,10-14$. Meanwhile, the two ubiquitin-like protein conjugation systems, the ATG5-ATG12 conjugation system and the ATG8 family proteins-phosphatidylethanolamine (PE) conjugation system, 
facilitate phagophore expansion and completion by catalyzing the PE-lipidation of ATG8 family proteins ${ }^{5,6,8,15}$. Specifically, in the ubiquitin-like protein conjugation systems, the ubiquitin-like ATG12 conjugates with ATG5 mediated by the E1-like ATG7 and E2-like ATG10, forming the ATG12 ATG5 conjugate, which can further associate with ATG16L1 to form the E3-like ATG12 ATG5-ATG16L1 complex for catalyzing the PE-conjugation of ATG8 family proteins ${ }^{6,8,15}$. Notably, the PE-conjugation of ATG8 family proteins plays a critical role in autophagic vesicle trafficking and autophagosome-lysosome fusion except for autophagosome biogenesis ${ }^{15-18}$. Although the core autophagic proteins are well demonstrated to be essential for canonical autophagy, many of the detailed molecular mechanisms underpinning the specific associations of these core autophagic proteins involved in the biogenesis of autophagosome remain elusive. Especially, the direct interaction between PI3P-binding WIPI2b and ATG16L1 is considered to bridge PI3P production and the PE-lipidation of ATG8 family proteins ${ }^{10}$, two crucial steps in autophagosome formation, but the related molecular mechanism is still largely unknown.

In addition to non-selective canonical autophagy, recent mounting studies have uncovered a large number of selective autophagy processes mediated by different types of autophagy receptors ${ }^{16,18-24}$. Currently identified autophagy receptors in mammals, such as P62, Optineurin, NDP52, TAX1BP1, NBR1, Nix, FUNDC1, STBD1, CCPG1, FAM134B, TEX264, RTN3, ATL3 and Sec62, all encompass a cargoassociating domain that can specifically recognize certain types of autophagic cargoes to be degraded as well as a unique LC3-interacting region (LIR) or ubiquitin-interacting motif (UIM) that can recruit the ATG8 family proteins known as LC3A, LC3B, LC3C, GABARAP, GABARAPL1 and GABARAPL2 in mammals ${ }^{19,20,25-27}$. During selective autophagy, after recognition of the targeting cargo, autophagy receptor can effectively induce in situ autophagosome formation to envelope and sequester the cargo by recruiting relevant core autophagic proteins ${ }^{18,20,24,25}$. Strikingly, recent studies showed that the autophagy receptor CCPG1, P62, NDP52 and TAXBP1 can directly interact with RB1CC1, thereby recruiting the ULK complex to induce autophagosome formation in close proximity to the targeting cargoes ${ }^{28-32}$. However, until now, the mechanistic bases underlying the in situ initiation of autophagosome formation by other autophagy receptors during selective autophagy remain unknown.

As a key autophagic protein, ATG16L1 contains an N-terminal ATG5-binding domain that is responsible for the interaction with ATG12 ATG5 conjugate to assemble the E3-like ATG12 ATG5-ATG16L1 complex ${ }^{33}$, and a following coiled-coil domain (Fig. 1a), which can induce the self-dimerization of ATG16L1 and associate with Rab33 ${ }^{34,35}$. In addition, the middle region of ATG16L1 contains a WIPI2bbinding motif as well as a FIR motif (Fig. 1a), which can interact with RB1CC1 for recruiting the ULK complex ${ }^{36,37}$. Unlike its yeast homolog ATG16, the C-terminal region of ATG16L1 contains an additional WD40 repeats domain (Fig. 1a), which can specifically recognize invading pathogens or pathogencontaining vacuoles by binding to relevant proteins including ubiquitin ${ }^{38}$, the complement protein $\mathrm{C} 3^{39,40}$, and V-ATPase ${ }^{41}$, thereby endowing ATG16L1 with specific functions in antibacterial selective autophagy (also called xenophagy) ${ }^{42}$. Notably, in addition to its essential role in canonical autophagy, the interaction between ATG16L1 and WIPI2b is also required for xenophagy ${ }^{10}$. Despite the crucial roles of ATG16L1 in 
xenophagy have been well established, the mechanistic bases underpinning the specific functions of ATG16L1 in selective autophagy are still poorly understood. How ATG16L1 interacts with RB1CC1 and whether ATG16L1 can be classified as an autophagy receptor remain to be elucidated.

In this study, we systemically characterized the interaction between ATG16L1 and WIPI2b, and elucidated the detailed interaction mechanism by solving their atomic complex structure. Furthermore, we discovered that in addition to interacting with the Claw domain of RB1CC1, ATG16L1 FIR can serve as an atypical LIR motif to selectively recognize mammalian ATG8 family proteins. Our determined crystal structures of the ATG16L1 FIR/RB1CC1 Claw complex and the ATG16L1 FIR/GABARAPL1 complex not only uncover the detailed molecular mechanism governing the specific interactions of ATG16L1 FIR with RB1CC1 and ATG8 family members, but also reveal that RB1CC1 and ATG8 family proteins are mutually exclusive in binding to ATG16L1 FIR. Taken together, our findings provide novel mechanistic insights into the distinct functions of ATG16L1 in canonical autophagy and selective autophagy.

\section{Results}

\section{Biochemical characterizations of the interaction between WIPI2b and ATG16L1}

To elucidate the molecular mechanism governing the interaction between WIPI2b and ATG16L 1, we firstly sought to biochemically characterize their interaction. However, after numerous trials, we failed to obtain soluble recombinant proteins of WIPI2b from E. Coil cells. Interestingly, a careful sequence alignment analysis of WIPI2b from different species together with relevant secondary structure predication and homology structure modelling by Phyre 2 server $^{43}$, revealed that there is a long loop insertion (residues 265-297) within the putative sixth blade of WIPI2b WD40 repeats domain (Supplementary Fig. 1a). Strikingly, this insertion region contains a large portion of hydrophobic residues, and importantly, previous studies of Hsv2, a yeast homolog of WIPI protein, demonstrated that the corresponding region of Hsv2 can penetrate the membrane to promote the membrane binding of Hsv2 ${ }^{44,45}$. Therefore, we wondered whether we can delete this hydrophobic loop region of WIPI2b to facilitate its folding in E. Coil cells. Thus, we designed a core WD40 construct of WIPI2b (hereafter referred to as WIPI2 ${ }^{\Delta}$ ), in which the putative membrane-insertion region of WD40 repeats domain (residues 265-297) as well as the predicated unstructured $\mathrm{N}$-terminal region (residues 1-12) and C-terminal region (residues 363-436) of WIPI2b was removed (Fig. 1b). Fortunately, using this WIPI $2 \mathrm{~b}^{\Delta}$ construct, we can readily obtain soluble proteins of WIPI2b from E. Coil cells, despite with a very lower expression level. Then, using these soluble WIPI $2 \mathrm{~b}^{\Delta}$ proteins, we sought to map out the precise WIPI2b-binding region of ATG16L1. Based on the sequence conservation of ATG16L1 as well as the fact that the residues 207-230 of ATG16L1 are essential for the specific interaction between ATG16L1 and WIPI2b in cells ${ }^{10}$, we chose four ATG16L 1 fragments (residues 186-236, 207-236, 213-236 and 221-236), and then purified these proteins and conducted quantitative isothermal titration calorimetry (ITC)-based analyses to measure their interactions with WIPI $2^{\Delta}$. Our ITC results revealed that the ATG16L1(186-236) and ATG16L1(207-236) fragments bind to WIPI2b ${ }^{\Delta}$ with similar Kd values (Supplementary Fig. 2a and Fig. 1c, d) while ATG16L1(213-236) shows a much 
reduced binding affinity towards WIPI2b $\mathrm{b}^{\Delta}$ compared with ATG16L1(186-236) or ATG16L1(207-236) (Supplementary Fig. 2b and Fig. 1c), and ATG16L1(221-236) even can't interact with WIPI2b ${ }^{\Delta}$ (Supplementary Fig. 2c and Fig. 1c). Collectively, we concluded that the WIPI2b-binding motif of ATG16L1 is located within the ATG16L1(207-236) fragment (hereafter referred to as ATG16L1 WBM).

\section{Overall Structure Of Wipi2b In Complex With Atg16l1 Wbm}

Then, using the purified WIPI2 $\mathrm{b}^{\Delta}$ and ATG16L1 WBM complex, we successfully obtained good crystals that diffracted to $1.50 \AA$ resolution. Using the molecular replacement method with the modified WD40 structure of yeast ATG18 (PDB ID: 5LTG), we determined the crystal structure of the WIPI2b ${ }^{\Delta} /$ ATG16L1 WBM complex (Supplementary Table 1). In the final complex structural model, an asymmetric unit contains one WIPI2b ${ }^{\Delta} /$ ATG16L1 WBM complex, which forms a 1:1 stoichiometric binary complex (Fig. 1e). As expected, the WIPI2b $\mathrm{b}^{\Delta}$ in the complex structure adopts a typical WD 40 repeats folding, featuring a seven-bladed $\beta$-propeller architecture (Fig. 2a), and the overall structure of WIPI2b in the WIPI2b/ATG16L1 complex is similar to that of the apo-form structures of Hsv2 and WIPI3 (Supplementary Fig. 3a, b). Intriguingly, the clearly defined WIPI2b-binding motif of ATG16L1 in the complex structure contains 23 highly conserved residues (residues 209-231) and mainly forms a continuous a-helix (Fig. 1e, 2a and Supplementary Fig. 2b), which packs extensively with a solventexposed groove between blade 2 and blade 3 of WIPI2b, burying a total of $\sim 544 \AA^{2}$ surface area (Fig. 1f). Further structural analysis showed that WIPI2b contains the signature "FRRG" motif, and forms two phosphoinositide-binding sites in the blade 5 and blade 6 region (Supplementary Fig. 3c, d), similar to other PROPPIN proteins ${ }^{44-46}$. Importantly, the phosphoinositide-binding sites and the ATG16L1-binding site are located in the opposite sides of the WD40 repeats domain of WIPI2b (Supplementary Fig. 3c, d), in accord with the fact that WIPI2b can associate with membrane and recruit the ATG12 ATG5-ATG16L1 complex simultaneously ${ }^{10}$. Notably, the deleted hydrophobic loop region of WIPI2b is far away from the ATG16L1-binding site (Fig. 1e). Therefore, it's unlikely to affect the interaction between WIPI2b and ATG16L1. Further structural comparison analyses revealed that although both WIPI2b and WIPI3 rely on a similar groove between blade 2 and blade 3 to recognize their binding partners, the overall binding mode of WIPI2b with ATG16L1 is completely different from that of WIPI3 and ATG2A interaction (Fig. 1e and Supplementary Fig. 3e, f).

\section{The Molecular Interface Of Wipi2b/atg16l1 Wbm Complex}

Further detailed structure analyses of the molecular interface of WIPI2b ${ }^{\Delta} /$ ATG 16 L1 WBM complex revealed that the ATG16L1 helix binds to a highly electropositive and hydrophobic groove between blades 2 and 3 of WIPI2b (Supplementary Fig. $4 a$ and Fig. 2b), and the specific interaction between WIPI2b and ATG16L1 is mainly mediated by extensive hydrophobic contacts and polar interactions (Fig. 2b, c and Supplementary Fig. 4a). Specifically, the hydrophobic side chains of L220, L224, A227 and A228 of ATG16L1 occupy a hydrophobic groove formed by the side chains of L64, L69, V83, 192, C93, 1124 and M127 from WIPI2b (Fig. 2c). Concurrently, the polar side chain group of Q217 and the backbone carboxyl group of A227 from ATG16L1 respectively interact with the K128, S68 and L69 residues of WIPI2b, 
forming three specific hydrogen bonds (Fig. 2c). In addition, the WIPI2b/ATG16L1 complex is further stabilized by a Glu-Lys pair (Glu226 ${ }_{\text {ATG16L1 }}$-Lys88 ${ }_{\text {WIPI2b }}$ ) and a Glu-Arg pair (Glu230 ${ }_{\text {ATG16L1 }}$-Arg108 WIPI2b $_{\text {}}$ ) of salt bridges (Fig. 2c and Supplementary Fig. 4a). In line with their important structural roles, all of these key residues of ATG16L1 and WIPI2b involved in the binding interface are strictly conserved across different eukaryotic species (Supplementary Fig. 1). Using ITC method, we further validated the specific interactions between ATG16L1 and WIPI2b observed in the complex structure. Consistent with our structural data, our ITC results showed that point mutations of key interface residues either from WIPI2b or ATG16L1, such as the L69A, 192Q, R108E mutations of WIPI2b or the L224Q, E226R, A227Q, E230R mutations of ATG16L1, all disrupted the interaction between ATG16L1(207-236) and WIPI2b ${ }^{\Delta}$ in our ITCbased assays (Supplementary Fig. 5, 6 and Fig. 1d). Importantly, in agreement with our ITC results, further co-immunoprecipitation assays showed that point mutations of key binding interface residues, including the 192Q, R108E mutations of WIPI2b and the L224Q, A227Q mutations of ATG16L1, all essentially abolish the specific association of full-length WIPI2b and ATG16L1 in cells (Fig. 2e, f). In contrast, the disease-associated WIPI2b V231M missense mutation ${ }^{47}$, which is located in the phosphoinositidebinding region of WIPI2b (Supplementary Fig. $4 b$ ), has a mild effect on the interaction of WIPI2b and ATG16L1 in vitro or in cells (Supplementary Fig. 5e and Fig. 2d, e).

\section{Biochemical characterizations of the interaction between ATG16L1 and RB1CC1 Claw domain}

In addition to binding WIPI2b, ATG16L1 can also directly interact with RB1CC $1^{36,37}$, a subunit of the ULK complex, which mainly contains an ATG13-binding N-terminal domain, a LIR motif followed by several coiled-coil regions and an extreme C-terminal Claw domain (Fig. 1a). Therefore, we also sought to elucidate the mechanistic bases underlying the interaction between ATG16L1 and RB1CC1. Previous studies uncovered a middle region including residues 229-250 of ATG16L1 is required for the interaction between ATG16L1 and RB1CC $1^{36,37}$. Strikingly, further detailed sequence alignment analysis of the RB1CC1-binding region of ATG16L1 with that of NAP1, SINTBAD and CCPG1, all of which were demonstrated to directly interact with RB1CC1 Claw domain ${ }^{28,30}$, revealed that ATG16L1 contains a putative RB1CC1 Claw-binding FIR motif within its RB1CC1-binding region (Fig. 3a), which has a characteristic core sequence $\psi \Theta x x \Gamma$ (where $\psi$ represents an acidic Asp, Glu or phosphorylated Ser/Thr residue, $\Theta$ represents a bulk hydrophobic Ile, Leu, Val or aromatic Phe, Tyr, Trp residue, $\Gamma$ being a hydrophobic Leu, lle or Val residue, and $x$ represents any residues) ${ }^{32}$. Using the analytical gel filtration chromatography-based analysis, we confirmed that the putative FIR region of ATG16L1 (residues 235247) can directly interact with RB1CC1 Claw domain (residues 1490-1594) (Fig. 3b). In contrast, the ATG16L1 fragment (residues 78-206) that contains the dimeric coiled-coil and the Rab33-binding region is unable to bind RB1CC1 Claw (Supplementary Fig. 7a). Further a quantitative fluorescent polarization (FP)-based assay revealed that the FITC-labeled ATG16L1 FIR peptide can specifically interact with RB1CC1 Claw with a Kd value of about $6.79 \mu \mathrm{M}$ (Fig. 3c).

\section{The Overall Structure Of The Atg16l1 Fir/rb1cc1 Claw Complex}


Then, we intended to determine the ATG16L1 FIR/RB1CC1 Claw complex structure to elucidate how ATG16L1 FIR interacts with the Claw domain of RB1CC1. After numerous trials, we finally managed to solve the crystal structure of ATG16L1 FIR in complex with RB1CC1 Claw at $1.61 \AA$ resolution (Supplementary Table 2). The determined ATG16L1 FIR/RB1CC1 Claw complex structure is composed of two ATG16L1 FIR molecules and a RB1CC1 Claw dimer, forming a unique 2:2 stoichiometric heterotetramer (Fig. 3d), in line with our multi-angle light scattering result that ATG16L1 and RB1CC1 Claw domain can form a stable binary complex with the same 2:2 binding stoichiometry in solution (Supplementary Fig. 7b). In the complex structure, except for a unique N-terminal a-helix that was only found in one of the two RB1CC1 Claw domains owing to crystal packing (Supplementary Fig. 8a), the two monomeric RB1CC1 Claw domains adopt a highly similar core architecture assembled by six $\beta$-strands and one a-helix (Fig. 3d and Supplementary Fig. 8b). In parallel, the two ATG16L1 FIR molecules in the complex structure mainly form two short $\beta$-strands that symmetrically augment the $\beta 4$-strands of two RB1CC1 Claw domains in an anti-parallel manner (Fig. 3d). Interestingly, further structural comparison analyses showed that the overall structure of the monomeric RB1CC1 Claw domain in the ATG16L1 FIR/RB1CC1 Claw complex is highly similar to that of the apo-form RB1CC1 Claw domain (PDB ID: 6DCE) (Supplementary Fig. 8c), and the binding of ATG16L1 FIR to RB1CC1 Claw can induce an obvious conformational rearrangement of the RB1CC1 Claw dimer (Supplementary Fig. 8d).

\section{The Binding Interface Between Atg16l1 Fir And Rb1cc1 Claw}

In the ATG16L1 FIR/RB1CC1 Claw complex structure, each ATG16L1 FIR molecule packs extensively with a highly electropositive and hydrophobic concave groove that is situated adjacent to the $\beta 4 / \beta 5$ connecting region of the monomeric RB1CC1 Claw domain, burying a total surface area of $\sim 505 \AA^{2}$ (Fig. 3d, e and Supplementary Fig. 9). Further careful analyses of the molecular interface in the ATG16L1 FIR/RB1CC1 Claw complex structure revealed that the hydrophobic side chain of ATG16L1 I240 deeply inserts into a hydrophobic pocket formed by the side chains of C1565, A1567, F1574 and F1582 residues of RB1CC1 Claw domain (Fig. 3f and Supplementary Fig. 9b), and concurrently, the hydrophobic side chains of ATG16L1 I243 and V244 residues have hydrophobic contacts with the aromatic side chain of Y1564 and the aliphatic side chain of K1581 from RB1CC1 (Fig. 3f and Supplementary Fig. 9b). Furthermore, the negatively charged D238, D239 and E241 residues of ATG16L1 FIR form specific hydrogen bonding and charge-charge interactions with the positively charged K1569, K1568 and K1562 residues of RB1CC1 Claw (Fig. 3e, f). In addition, the backbone groups of E241, I243 and V244 residues of ATG16L1 FIR couple with the backbone groups of Q1566 and Y1564 residues of RB1CC1 to form five specific backbone hydrogen bonds (Fig. 3e, f). In agreement with their important structural roles, all of these key binding interface residues of ATG16L1 and RB1CC1 are highly conserved in different eukaryotic species (Supplementary Figs. 1 and 10). Consistent with our aforementioned structural results, further FPbased assays showed that point mutations of key interface residues of RB1CC1 Claw, such as the Y1568A, K1569A, R1573E and F1574Q mutations, all significantly decrease the specific interaction of RB1CC1 Claw with ATG16L1 FIR (Supplementary Fig. 11 and Fig. 3g). Reciprocally, point mutations of key binding interface residues from ATG16L1 FIR, including the D238R, D239R, I240Q, E241R and I243Q 
mutations of ATG16L1 FIR, all largely attenuate or essentially disrupt the interaction between RB1CC1 Claw and ATG16L1 FIR (Supplementary Fig. 12 and Fig. 3h). Therefore, all those mutagenesis-based biochemical assays confirmed the specific interactions between ATG16L1 FIR and RB1CC1 Claw observed in the solved complex structure.

\section{The FIR motif of ATG16L1 can selectively bind to mammalian ATG8 family proteins}

Since the FIR motif of ATG16L1 has a high similarity with the CCPG1 FIR motifs (Fig. 3a), which was recently proved to directly recognize mammalian ATG8 family proteins ${ }^{32}$, we suspected ATG16L1 FIR may also have the ability to recognize mammalian ATG8 orthologs. Indeed, using analytical gel filtration chromatography-based assays, we demonstrated that the FIR motif of ATG16L1 can directly interact with six mammalian ATG8 members (Fig. 4a and Supplementary Fig. 13). Interestingly, further ITC analyses revealed that the FIR motif of ATG16L1 preferentially binds to GABARAPL1 and LC3C with a binding affinity $K \mathrm{~d}$ value of $\sim 4.59 \mu \mathrm{M}$ and $\sim 6.27 \mu \mathrm{M}$, respectively (Fig. 4b, i and Supplementary Fig. 14). Then, we chose GABARAPL1 as a representative to further characterize its interaction with ATG16L1. Our additional ITC-based analyses using three different fragments of ATG16L1 confirmed that the FIR region of ATG16L1 (residues 235-247) is sufficient for binding to ATG8 family proteins (Fig. 4c, $d$ and Supplementary Fig. 15a, b). Meanwhile, we also utilized NMR to further validate the interaction between GABARAPL1 and ATG16L1. Titrations of ${ }^{15} \mathrm{~N}$-labelled GABARAPL1 with un-labelled ATG16L1(235-247) or ATG16L1(78-247) proteins showed that many peaks in the ${ }^{1} \mathrm{H}^{-15} \mathrm{~N}$ HSQC spectrum of GABARAPL1 undergo significant chemical shift changes or peak-broadenings (Fig. 4d and Supplementary Fig. 15c), confirming that the FIR motif-containing ATG16L1 fragments can directly bind to GABARAPL1. Taken together, all these data clearly demonstrated that the FIR region of ATG16L1 can serve as an unconventional LIR motif to selectively interact with six mammalian ATG8 orthologs.

\section{The Structure Of Atg16l1 Fir In Complex With Gabarapl1}

To further uncover the detailed molecular mechanism underlying the selective recognitions of ATG8 family proteins by ATG16L1 FIR, we determined the high-resolution crystal structure of GABARAPLI in complex with ATG16L1 FIR (Supplementary Table 3). In the determined GABARAPL1/ATG16L1 FIR complex structure, GABARAPL1 adopts a typical ATG8 family protein architecture that is mainly composed of a ubiquitin-like structural core and two preceding N-terminal a-helices (Fig. 4e). In the complex structure, the clearly defined ATG16L1 FIR motif contains 10 highly conserved residues spanning from Q236 to D245 of ATG16L1 (Fig. 4e and Supplementary Fig. 16a), and adopts an extended configuration to occupy the canonical LIR-binding groove of GABARAPL1, which is mainly formed by the $\beta 1$-strand, $\beta 2$-strand, a2-helix and a3-helix of GABARAPL1 (Fig. 4e-h). Further structural analyses of the binding interface of the GABARAPL1/ATG16L1 FIR complex showed that the interaction between GABARAPL1 and ATG16L1 FIR is mainly mediated by both polar and hydrophobic interactions (Fig. 4f-h). In particular, the negatively charged side chains of ATG16L1 D239, E241 interact with the positively charged side chains of K48, K46 and R67 of GABARAPL1 to form three charge-charge interactions, and the backbone amide and oxygen groups of ATG16L1 E241 together with the backbone amide group of 
ATG16L1 I243 form three backbone hydrogen bonds with the GABARAPL1 K48 and L50 residues (Fig. 4f and $\mathbf{h}$ ). In addition, the GABARAPL1/ATG16L1 FIR interaction is further strengthened by four specific hydrogen bonds, two of which are formed between the backbone oxygen groups of D238 and I240 residues of ATG16L1 and the positively charged side chain of GABARAPL1 K46, while the other two are mediated by the backbone oxygen group of ATG16L 1 I 243 and the positively charged side chain of GABARAPL1 R28 (Fig. 4h). In parallel, the hydrophobic side chains of ATG16L1 I240 and V242 residues pack against a hydrophobic groove of GABARAPL1 formed by the hydrophobic side chains of I21, P30, L50, F104 and the aliphatic portion of the side chain of GABARAPL1 K48 residue (Fig. $4 \mathrm{~g}$ and $\mathbf{~ h}$ ). Meanwhile, the hydrophobic side chain of ATG16L1 I243 occupies a hydrophobic pocket assembled by the side chains of Y49, V51, P52, L55, F60 and L63 residues of GABARAPL1 (Fig. $4 \mathrm{~g}$ and $\mathbf{h}$ ). Consistently, all these key binding interface residues of GABARAPL1 and ATG16L1 FIR are highly conserved during evolution (Supplementary Fig. 1b and 17). Using ITC-based assays, we further verified the specific interaction between GABARAPL1 and ATG16L1 FIR. Individual point mutations of key residues involved in the binding interface of GABARAPL1/ATG16L1 FIR complex either from GABARAPL1 or ATG16L1 FIR, such as the 121Q, K48E, L50Q and R67E mutations of GABARAPL1 (Supplementary Fig. 18 and Fig. 4i) or the D239R, I240Q, E241R V242E and I243Q mutations of ATG16L1 FIR (Supplementary Fig. 19 and Fig. 4i), all dramatically decreased or essentially disrupted the association of GABARAPL1 with ATG16L1 FIR. Notably, further careful sequence and structure comparison analyses revealed that the amino acid sequence pattern of ATG16L1 FIR is highly similar to that of the unconventional LIR motif of autophagy receptor NDP52 (Supplementary Fig. 15b), and the overall binding mode of ATG16L1 FIR motif with GABARAPL1 resembles that of the atypical NDP52 LIR motif and LC3C interaction observed in the NDP52/LC3C complex structure (PDB ID: 3VVW) (Fig. 4f-h and Supplementary Fig. 16c-e).

\section{The relationships of RB1CC1, GABARAPL1 and WIPI2b in binding to ATG16L1}

Intriguingly, our structural analyses revealed that ATG16L1 FIR motif adopts essentially the same key residues to recognize RB1CC1 Claw domain and GABARAPL1, such as the D239, I240, E241 and I243 residues (Figs. 3f, 4h and Supplementary Fig. 1b). Thus, RB1CC1 and ATG8 family proteins should be mutually exclusive in binding to ATG16L1 FIR. As expected, further analytical gel filtration chromatography coupled with SDS-PAGE assays demonstrated that the ATG16L1 FIR/RB1CC1 Claw binary complex is unable to recruit GABARAPL1 (Fig. 5a). Therefore, like that of P62 and Optineurin 29,32, ATG16L1 alone is unlikely to simultaneously recruit ULK complex and ATG8 family proteins. Given that ATG16L1 FIR motif is C-terminally adjacent to the WIPI2b-binding motif of ATG16L1 (Supplementary Fig. 1b), we also test the relationship between WIPI2b and RB1CC1 or GABARAPL1 in binding to ATG16L1. Using analytical gel filtration chromatography coupled with SDS-PAGE analyses, we revealed that RB1CC1 Claw, but not GABARAPL1, can form a stable ternary complex with WIPI2b ${ }^{\Delta}$ and ATG16L1(207-247) that contains the WIPI2b-binding site as well as the RB1CC1-binding FIR motif (Fig. $5 \mathrm{~b}$ and d). These biochemical results clearly demonstrated that WIPI2b and RB1CC1 can simultaneously bind to ATG16L1, consistent with a previous study ${ }^{10}$, while WIPI2b and ATG8 family protein, such as GABARAPL1, are competitive in binding to ATG16L1, likely due to the potential steric hindrance. 


\section{Discussion}

Previous functional studies demonstrated that the four mammalian WIPI proteins can be classified into two sub-groups: the WIPI1/2 group and the WIPI3/4 group, which can specifically recognize ATG16L1

and ATG2A/B, respectively ${ }^{10-12,14}$. The molecular mechanism governing the selective interaction of WIPI3/4 with ATG2 has been well elucidated by a recent elegant structural study ${ }^{48}$. In this study, we determined the WIPI2b/ATG16L1 complex structure, and uncovered the detailed binding mechanism as well as the key determinants for the specific interaction between WIPI2b and ATG16L1 (Fig. 2). Importantly, further structure-based sequence alignment analysis revealed that the key ATG16L1-binding interface residues of WIPI2b can be also found in its close homologue WIPI1, but diverge from WIPI3 and WIPI4 (Supplementary Fig. 20). Thus, our work may also provide a mechanistic explanation to the different autophagic functions mediated by the WIPI1/2 group and the WIPI3/4 group. Interestingly, although our sequence alignment analysis showed that WIPI1 and WIPI2 share the identical interface residues for binding to ATG16L1 (Supplementary Fig. 20), a previous study clearly demonstrated that WIPI1 has a much weaker interaction with ATG16L1 compared with WIPI2b ${ }^{10}$. Unfortunately, we are unable to obtain soluble WIPI1 proteins, thereby preventing detailed biochemical and structural characterizations of the interaction between WIPI1 and ATG16L1. Apparently, additional studies are required to elucidate the detailed mechanism governing the weak interaction of WIPI1 with ATG16L1 as well as the potential relevant regulative mechanism.

In the autophagy field, to define a protein as an autophagy receptor to mediate selective autophagy, it must have three key characteristics. Firstly, it must have the ability to recognize prospective autophagic cargoes. Then, after recognition of the targeting cargoes, it should be able to recruit upstream autophagic machine, such as the ULK complex, to promote the initiation and elongation of phagophores for the engulfment of the targeting cargoes. In addition, it also should have a LIR or UIM motif to interact with ATG8 family proteins for anchoring autophagic cargoes around nascent phagophores. Previous functional studies have well established that mammalian ATG16L 1 can function as an adaptor to specifically recognize invading pathogens or pathogen-containing vacuoles through its C-terminal WD40 repeats domain to mediate xenophagy processes ${ }^{38,39,41,42}$. Meanwhile, ATG16L1 can directly interact with RB1CC1 to recruit the ULK complex ${ }^{36,37}$. However, due to the lack of conventional ATG8-binding motif, whether ATG16L1 can be classified as an autophagy receptor remains elusive. In this work, we uncovered that the FIR region of ATG16L1 contains an atypical LIR motif, which can directly interact with mammalian ATG8 orthologs (Fig. 4). Therefore, based on our results together with other groups' reports, we can surely define ATG16L1 as a mammalian autophagy receptor involved in xenophagy. Interestingly, a missense T300A mutation of ATG16L1 that locates in the middle loop region between the FIR motif and WD40 repeats domain is strongly associated with the incidence of Crohn's disease ${ }^{49}$, a type of inflammatory bowel disease mainly caused by the defect in xenophagy. Importantly, this T300A polymorphism can dramatically increase caspase 3-mediated cleavage of ATG16L1, resulting in lower levels of intact ATG16LI protein as well as decreased xenophagy in cells ${ }^{50,51}$. Based on our studies, the T300 residue is located between the RB1CC1/ATG8-binding FIR motif and the cargo-recognizing WD40 
repeats domain of ATG16L1. Apparently, the cleavage of ATG16L1 T300A mutant around this T300A site by caspase 3 should completely disrupt the function of ATG16L1 to serve as an autophagy receptor for mediating xenophagy. Therefore, our studies may provide a mechanistic explanation to why the T300A mutation of ATG16L1 can cause Crohn's disease.

Interestingly, our quantitative analyses of the interactions of ATG16L1 FIR with different mammalian ATG8 family members revealed that ATG16L1 FIR can selectively bind to six mammalian ATG8s, and preferentially bind to GABARAPL1 and LC3C (Fig. 4b, i and Supplementary Fig. 14). Intriguingly, detailed sequence alignment analysis elucidated that several key interface residues for interacting with ATG16L1 are quite different among six mammalian ATG8 family proteins (Supplementary Fig. 21). For example, the residue corresponding to the R28 residue of GABARAPL1 that forms two hydrogen bonds with the backbone oxygen group of ATG16L1 I243 is a Lys residue in the LC3 subfamily, and the residues corresponding to the bulk hydrophobic L55 and F60 residues in GABARAPL1 are two much smaller Val and Leu in LC3A and LC3B (Supplementary Fig. 21b). The identification of these non-conserved interface residues among different ATG8 family proteins may rationalize the selective recognition of ATG16L1 FIR towards different mammalian ATG8 orthologs. In the future, it will be interesting to know whether different mammalian ATG8 orthologs play redundant or specific roles in ATG16L1-mediated selective autophagy processes.

Finally, we proposed a model to depict the two distinct functions of ATG16L1 in canonical autophagy and xenophagy (Fig. 5d). In this model, ATG16L1 always associates with the ATG12 ATG5 conjugate, forming the ATG12 ATG5-ATG16L1 complex to conduct its ATG8-conjugating functions (Fig. 5d). In the canonical autophagy process, the ATG12 ATG5-ATG16L1 complex mainly functions as the downstream effector of WIPI2b that can directly sense the PI3P generated by the upstream PI3KC3-C1 complex, and is specifically recruited to the nascent preautophagosomal membrane mediated by the direct interaction between WIPI2b and ATG16L1 as uncovered in this study. Subsequently, the recruited ATG12 ATG5-ATG16L1 complex can act as an E3-like enzyme to facilitate the PE-conjugation of ATG8 family proteins on the phagophore membrane (Fig. 5d). Eventually, the enriched PE-conjugated ATG8 family proteins might compete with WIPI2b for binding to ATG16L1, and ultimately promote the expansion and closure of the phagophore membrane to form the autophagosome (Fig. $5 \mathrm{~d}$ ). On the other hand, ATG16L1 could serve as an autophagy receptor in xenophagy to specifically recognize the ubiquitin and/or C3 proteins decorated on the invading pathogen through its C-terminal WD40 repeats domain (Fig. 5d). After that, ATG16L1 could recruit the RB1CC1-containing ULK complex mediated by the specific interaction between ATG16L1 FIR and RB1CC1 Claw (Fig. 5d). Meanwhile, ATG16L1 might also engage with WIPI2b, which in turn can recruit the PI3KC3-C1 complex (Fig. 5d). Subsequently, the activated ULK complex and/or PI3KC3-C1 complex could in situ initiate autophagosome formation by promoting the recruitment of downstream autophagic machinery, and eventually lead to the PE-lipidation and the enrichment of ATG8 family proteins on the phagophore membrane (Fig. 5d). Then, the enriched PEmodified ATG8 family proteins could facilitate the formation of autophagosome around the targeting pathogen by ATG16L1 (Fig. 5d), and in parallel, might release RB1CC1 and WIPI2b from binding to ATG16L1 for further recycling (Fig. 5d). 


\section{Materials And Methods}

Materials. HEK293T cell lines were kindly provided by Prof. Junying Yuan from Interdisciplinary Research Center on Biology and Chemistry, CAS, Shanghai, China. The full length human WIPI2b, ATG16L1 and RB1CC1 were obtained from Prof. Jiahuai Han from School of Life Sciences, Xiamen University, Xiamen, China. The synthetic peptide EQDDDIEVIVDET (ATG16L1 FIR) was purchased from the China Peptides company and the purities of the commercially synthesized peptides were $>98 \%$.

Protein expression and purification. The DNA fragment encoding human RB1CC1 (residues 1490-1594) were cloned into both pSUMO vector (a modified version of pET-28a vector containing a N-terminal Trx and $\mathrm{His}_{6}$-tag) and pET-32M-3C vector (a modified version of pET-32a vector containing a N-terminal Trx and $\mathrm{His}_{6}$-tag). The DNA fragment encoding WIPI2b (residues 13-362 without 265-297) were cloned into both pET-32a-SUMO vector (a modified version of pET-32a vector containing a $\mathrm{N}$-terminal $\mathrm{His}_{6}$ and SUMO tag) and the pRSF-Trx1-3C vector (a modified version of pRSF vector containing a N-terminal Trx and $\mathrm{His}_{6}$-tag). The DNA fragment encoding ATG16L1 (residues 186-236, 207-236, 213-236, 221-236, 225$236,235-247,78-247,78-206,207-247$ and 230-247) were all cloned into pACYC-Trx1-3C vector (a modified version of pACYC vector containing a N-terminal Trx and $\mathrm{His}_{6}$-tag). Meanwhile, the DNA fragments encoding six human ATG8s and mutants were all cloned into pET-32M-3C vector. For Coimmunoprecipitation assays, the DNA fragments encoding human full-length ATG16L1 and WIPI2b were separately cloned into pFlag-CMV-2 vector and pEGFP-C1 vector. All point mutations of WIPI2b, RB1CC1, GABARAPL1 and ATG16L1 used in this study were generated through standard PCR-based mutagenesis method and further confirmed by DNA sequencing.

Recombinant proteins were all expressed in BL21 (DE3) E. coli cells induced by $100 \mu \mathrm{M} \mathrm{IPTG}$ at $16^{\circ} \mathrm{C}$. The bacterial cell pellets were re-suspended in the binding buffer ( $50 \mathrm{mM}$ Tris, $500 \mathrm{mM} \mathrm{NaCl}, 5 \mathrm{mM}$ imidazole at $\mathrm{pH}$ 7.9), and then lysed by the ultrahigh pressure homogenizer FB-110XNANO homogenizer machine (Shanghai Litu Machinery Equipment Engineering Co., Ltd.). Then the lysis was spun down by centrifuge at $17000 \mathrm{rpm}(35000 \mathrm{~g})$ for 30 minutes to remove the pellets fractions. $\mathrm{His}_{6}$-tagged proteins were purified by $\mathrm{Ni}^{2+}-\mathrm{NTA}$ agarose (GE Healthcare) affinity chromatography. Each recombinant protein was further purified by size-exclusion chromatography (Superdex 75 or 200 26/60 column; GE Healthcare) equilibrated with the column buffer containing $20 \mathrm{mM}$ Tris, $100 \mathrm{mM} \mathrm{NaCl}, 1 \mathrm{mM}$ DTT and $1 \mathrm{mM}$ EDTA at $\mathrm{pH}$ 7.5. To obtain the WIPI2b/ATG16L1 complex used for crystallization, Trx-WIPI2b ${ }^{\Delta}$ and TrxATG16L1(207-236) were co-expressed in BL21 (DE3) E. coli cells. In the same way, RB1CC1/ATG16L1 complex came from the co-expression of Trx-RB1CC1(1490-1594) and Trx-ATG16L1(235-247). The Nterminal Trx tag was cleaved by $3 \mathrm{C}$ protease and removed by HisTrap excel column (GE Healthcare). Finally, these two complexes were further purified through Superdex 75 column equilibrated with the aforementioned column buffer. Special for six human ATG8s, their Trx tags were removed by MonoQ 10/10 ion-exchange column (GE Healthcare). Meanwhile, uniformly ${ }^{15} \mathrm{~N}$-labelled GABARAPL 1 proteins were prepared by growing bacteria in $\mathrm{M} 9$ minimal medium using ${ }^{15} \mathrm{NH}_{4} \mathrm{Cl}$ (Cambridge Isotope Laboratories Inc.) as the sole nitrogen source. 
Analytical gel filtration chromatography. Purified proteins were loaded on to a Superdex 200 increase 10/300 GL column (GE Healthcare) equilibrated with the same column buffer. Analytical gel filtration chromatography was carried out on an AKTA FPLC system (GE Healthcare). The results were further fitted through Origin 8.5 software.

NMR spectroscopy. The ${ }^{15} \mathrm{~N}$-labelled protein samples for NMR titration experiments were concentrated to $\sim 0.1 \mathrm{mM}$. All the protein samples for NMR studies were prepared in the $50 \mathrm{mM}$ potassium phosphate buffer containing $100 \mathrm{mM} \mathrm{NaCl}$ and $1 \mathrm{mM} \mathrm{DTT}$ at $\mathrm{pH} 6.5$, and NMR spectra were acquired at $25^{\circ} \mathrm{C}$ on an Agilent $800 \mathrm{MHz}$ spectrometer equipped with an actively z gradient shielded triple resonance cryogenic probe at the Shanghai Institute of Organic Chemistry.

Multi-angle light scattering. For multi-angle light-scattering measurement, RB1CC1(14901594)/ATG16L1(78-247) complex samples were injected into an AKTA FPLC system (GE Healthcare) with a Superdex 200 increase 10/300 GL column (GE Healthcare) with the same column buffer mentioned before. The chromatography system was coupled to a static light scattering detector (miniDawn, Wyatt Technology) and a differential refractive index detector (Optilab, Wyatt Technology). Data were collected every $0.5 \mathrm{~s}$ with a flow rate of $0.5 \mathrm{~mL} / \mathrm{min}$. Data were analyzed using the ASTRA 6 software (Wyatt Technology) and drawn on the Origin 8.5 software.

Fluorescence polarization assay. Fluorescence anisotropy binding assays were performed on the SpectraMax i3x Multi-Mode Detection Platform from Molecular Devices, using a $485 \mathrm{~nm}$ excitation filter and a $535 \mathrm{~nm}$ emission filter. Peptides were labeled with fluorescein isothiocyanate isomer I (SigmaAldrich) at their N-terminals. In this assay, the FITC-labeled peptide $(\sim 0.3 \mu \mathrm{M})$ was titrated with increasing amount of testing proteins in the column buffer at $25^{\circ} \mathrm{C}$. The $K \mathrm{~d}$ values were obtained by fitting the titration curves with the classical one-site binding model using GraphPad Prism 6 software.

Protein crystallization and structural elucidation. Crystals of the WIPI2b(13-362) ${ }^{\Delta(265-}$ 297)/ATG16L1(207-236) complex, the RB1CC1(1490-1594)/ATG16L1(235-247) complex and the GABARAPL1/ATG16L1(235-247) complex were all obtained using the sitting-drop vapor-diffusion method at $16^{\circ} \mathrm{C}$. The purified WIPI2b(13-362) $)^{\Delta(265-297)} /$ ATG16L1(207-236) complex $(20 \mathrm{mg} / \mathrm{mL})$ was mixed with equal volumes of reservoir solution containing $30 \% \mathrm{w} / \mathrm{v}$ Pentaerythritol ethoxylate (15/4 $\mathrm{EO} / \mathrm{OH}$ ), $100 \mathrm{mM}$ MES (pH 6.5) and $50 \mathrm{mM}$ Magnesium chloride. While, the crystal-growing condition of the RB1CC1(1490-1594)/ATG16L1(235-247) complex (38 mg/mL) was 10\% v/v 2-Propanol, $0.1 \mathrm{M}$ BICINE (pH 8.5), 30\% w/v Polyethylene glycol 1500. As for the GABARAPL1/ATG16L1(235-247) complex, the purified GABARAPL1 protein $(27 \mathrm{mg} / \mathrm{mL})$ was saturated with ATG16L1 FIR peptide with a molar ratio up to $1: 10$. Crystals were observed in the condition containing $0.1 \mathrm{M}$ Sodium cacodylate (pH 6.5), 40\% v/v MPD and $5 \% \mathrm{w} / \mathrm{v}$ PEG 8000. Before diffraction experiments, glycerol was added as the cryo-protectant. A $1.50 \AA$ resolution X-ray data set for the WIPI2b(13-362) ${ }^{\Delta(265-297)} /$ ATG16L1(207-236) complex, a $1.61 \AA$ resolution X-ray data set for the RB1CC1(1490-1594)/ATG16L1(235-247) complex and a $1.54 \AA$ resolution X-ray data set for the GABARAPL1/ATG16L1(235-247) complex were collected at the 
beamline BL17U1 or BL19U1 of the Shanghai Synchrotron Radiation Facility ${ }^{52}$. The diffraction data were processed upon HKL2000 53 or autoPROC ${ }^{54}$.

The phase problems of the WIPI2b/ATG16L1 complex, the RB1CC1/ATG16L1 complex and the GABARAPL1/ATG16L1 complex were all solved by molecular replacement method by using the ATG18 structure (PDB ID: 5LTG), the RB1CC1 Claw structure (PDB ID: 6DCE) and the GABARAPL1 structure (PDB ID: $5 \mathrm{LXI}$ ) respectively as the search model with $\mathrm{PHASER}^{55}$. The initial structural models were rebuilt manually using $\mathrm{COOT}^{56}$, and then refined through PHENIX ${ }^{57}$. Further manual model building and adjustments were completed via $\mathrm{COOT}^{56}$. The qualities of the final models were validated by MolProbity ${ }^{58}$. The final refinement statistics of solved structures in this study were listed in Supplementary Table 1, Table 2 and Table 3. All the structural diagrams were prepared using the program PyMOL (http://www.pymol.org/).

Co-immunoprecipitation. EGFP-tagged WIPI2b and Flag-tagged ATG16L1 plasmids (wild-type or mutants) were co-transfected into HEK293T cells using Lipofectamine 2000 transfection reagent (Thermo Fisher Scientific). Cells were collected $48 \mathrm{~h}$ after transfection and lysed in ice-cold cell lysis buffer ( $50 \mathrm{mM}$ Tris, $150 \mathrm{mM} \mathrm{NaCl}, 0.5 \% \mathrm{NP}-40,1 \mathrm{mM}$ PMSF, $1 \%$ protease inhibitor cocktail at $\mathrm{pH} 7.5$ ) for $40 \mathrm{~min}$ at $4^{\circ} \mathrm{C}$. Lysates were centrifuged at $14500 \mathrm{~g}$ for $15 \mathrm{~min}$ at $4^{\circ} \mathrm{C}$ to separate soluble fractions and cell debris. Supernatants were applied to anti-GFP mAb-Agarose (Medical \& Biological Laboratories) and incubated for $1 \mathrm{~h}$ at $4^{\circ} \mathrm{C}$. The beads and non-bound proteins were separated by centrifugation at $800 \mathrm{~g}$ for $3 \mathrm{~min}$ at $4^{\circ} \mathrm{C}$. After washing three times with cold wash buffer $(50 \mathrm{mM}$ Tris, $150 \mathrm{mM} \mathrm{NaCl}$ and $0.1 \% \mathrm{NP}-40$ at pH 7.5), the beads were re-suspended with the SDS-PAGE sample buffer and boiled at $100^{\circ} \mathrm{C}$. The prepared samples were analyzed by SDS-PAGE. The EGFP-tagged WIPI2b and Flag-tagged ATG16L1 were detected by western blot using the anti-GFP (Proteintech, 1:2000 dilution) and anti-Flag (Sigma-Aldrich, 1:2000 dilution) primary antibodies, respectively, and then the goat anti-mouse secondary antibody (Thermo Fisher Scientific, 1:10000 dilution).

\section{Declarations}

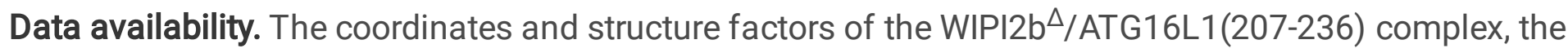
RB1CC1 Claw/ATG16L1 FIR complex and the GABARAPL1/ATG16L1 FIR complex solved in this study have been deposited in the Protein Data Bank under the accession code 7F69, 7F6A and 7F6B, respectively. All additional experimental data are available from the corresponding author on request.

\section{Acknowledgements}

We thank SSRF BL19U1 and BL17U for X-ray beam time, Prof. Jiahuai Han for the full length WIPI2b, RB1CC1 and ATG16L1 CDNA. This work was supported by grants from the National Natural Science Foundation of China $(21822705,32071219,91753113,21621002)$, the National Key R\&D Program of China (2016YFA0501903), the Science and Technology Commission of Shanghai Municipality (20XD1425200, 17JC1405200), the Strategic Priority Research Program of the Chinese Academy of 
Sciences (XDB20000000) (to L.P.); and grant from National Natural Science Foundation of China (31800646) (to Y.W.).

\section{Author contributions}

X.G. and L.P. designed research; Y.W., Y.T., J.L., Y.L., Y.W., X.X., M.Z., M.L., Y.Z., and L.S. performed research; X.G., J.L. and L.P. analyzed data; X.G. and L.P. wrote the paper.

\section{Competing interests}

The authors declare no competing interests.

\section{References}

1. Klionsky, D.J. \& Emr, S.D. Cell biology - Autophagy as a regulated pathway of cellular degradation. Science 290, 1717-1721 (2000).

2. Levine, B., Mizushima, N. \& Virgin, H.W. Autophagy in immunity and inflammation. Nature 469, 323-335 (2011).

3. Klionsky, D.J. et al. Guidelines for the use and interpretation of assays for monitoring autophagy (4th edition). Autophagy 17, 1-382 (2021).

4. Levine, B. \& Kroemer, G. Biological Functions of Autophagy Genes: A Disease Perspective. Cell 176, $11-42(2019)$.

5. Lamb, C.A., Yoshimori, T. \& Tooze, S.A. The autophagosome: origins unknown, biogenesis complex. Nat Rev Mol Cell Biol 14, 759-74 (2013).

6. Mizushima, N., Yoshimori, T. \& Ohsumi, Y. The role of Atg proteins in autophagosome formation. Annu Rev Cell Dev Biol 27, 107-32 (2011).

7. Yang, Z. \& Klionsky, D.J. Eaten alive: a history of macroautophagy. Nat Cell Bio/ 12, 814-22 (2010).

8. Feng, Y.C., He, D., Yao, Z.Y. \& Klionsky, D.J. The machinery of macroautophagy. Cell Research 24, 24-41 (2014).

9. Hurley, J.H. \& Young, L.N. Mechanisms of Autophagy Initiation. Annu Rev Biochem 86, 225-244 (2017).

10. Dooley, H.C. et al. WIPI2 links LC3 conjugation with PI3P, autophagosome formation, and pathogen clearance by recruiting Atg12-5-16L1. Mol Cell 55, 238-52 (2014).

11. Zheng, J.X. et al. Architecture of the ATG2B-WDR45 complex and an aromatic Y/HF motif crucial for complex formation. Autophagy 13, 1870-1883 (2017). 
12. Chowdhury, S. et al. Insights into autophagosome biogenesis from structural and biochemical analyses of the ATG2A-WIPI4 complex. Proceedings of the National Academy of Sciences of the United States of America 115, E9792-E9801 (2018).

13. Fracchiolla, D., Chang, C., Hurley, J.H. \& Martens, S. A PI3K-WIPI2 positive feedback loop allosterically activates LC3 lipidation in autophagy. J Cell Bio/ 219(2020).

14. Polson, H.E. et al. Mammalian Atg18 (WIPI2) localizes to omegasome-anchored phagophores and positively regulates LC3 lipidation. Autophagy 6, 506-22 (2010).

15. Geng, J. \& Klionsky, D.J. The Atg8 and Atg12 ubiquitin-like conjugation systems in macroautophagy. 'Protein modifications: beyond the usual suspects' review series. EMBO Rep 9, 859-64 (2008).

16. Cheng, X.F. et al. Structural basis of FYCO1 and MAP1LC3A interaction reveals a novel binding mode for Atg8-family proteins. Autophagy 12, 1330-1339 (2016).

17. Nguyen, T.N. et al. Atg8 family LC3/GABARAP proteins are crucial for autophagosome-lysosome fusion but not autophagosome formation during PINK1/Parkin mitophagy and starvation. J Cell Bio/ 215, 857-874 (2016).

18. Stolz, A., Ernst, A. \& Dikic, I. Cargo recognition and trafficking in selective autophagy. Nat Cell Biol 16, 495-501 (2014).

19. Johansen, T. \& Lamark, T. Selective autophagy mediated by autophagic adapter proteins. Autophagy 7, 279-96 (2011).

20. Rogov, V., Dotsch, V., Johansen, T. \& Kirkin, V. Interactions between Autophagy Receptors and Ubiquitin-like Proteins Form the Molecular Basis for Selective Autophagy. Molecular Cel/ 53, 167-178 (2014).

21. Gatica, D., Lahiri, V. \& Klionsky, D.J. Cargo recognition and degradation by selective autophagy. Nat Cell Biol 20, 233-242 (2018).

22. Green, D.R. \& Levine, B. To Be or Not to Be? How Selective Autophagy and Cell Death Govern Cell Fate. Cell 157, 65-75 (2014).

23. Kirkin, V. History of the Selective Autophagy Research: How Did It Begin and Where Does It Stand Today? J Mol Biol 432, 3-27 (2020).

24. Farre, J.C. \& Subramani, S. Mechanistic insights into selective autophagy pathways: lessons from yeast. Nat Rev Mol Cell Biol 17, 537-52 (2016). 
25. Kirkin, V. \& Rogov, V.V. A Diversity of Selective Autophagy Receptors Determines the Specificity of the Autophagy Pathway. Mol Cell 76, 268-285 (2019).

26. Johansen, T. \& Lamark, T. Selective Autophagy: ATG8 Family Proteins, LIR Motifs and Cargo Receptors. J Mol Bio/ 432, 80-103 (2020).

27. Marshall, R.S., Hua, Z., Mali, S., McLoughlin, F. \& Vierstra, R.D. ATG8-Binding UIM Proteins Define a New Class of Autophagy Adaptors and Receptors. Cell 177, 766-781 e24 (2019).

28. Smith, M.D. et al. CCPG1 Is a Non-canonical Autophagy Cargo Receptor Essential for ER-Phagy and Pancreatic ER Proteostasis. Developmental Cell 44, 217-+ (2018).

29. Turco, E. et al. FIP200 Claw Domain Binding to p62 Promotes Autophagosome Formation at Ubiquitin Condensates. Molecular Cell 74, 330-+ (2019).

30. Ravenhill, B.J. et al. The Cargo Receptor NDP52 Initiates Selective Autophagy by Recruiting the ULK Complex to Cytosol-Invading Bacteria. Molecular Cell 74, 320-+ (2019).

31. Vargas, J.N.S. et al. Spatiotemporal Control of ULK1 Activation by NDP52 and TBK1 during Selective Autophagy. Molecular Cell 74, 347-+ (2019).

32. Zhou, Z. et al. Phosphorylation regulates the binding of autophagy receptors to FIP200 Claw domain for selective autophagy initiation. Nat Commun 12, 1570 (2021).

33. Otomo, C., Metlagel, Z., Takaesu, G. \& Otomo, T. Structure of the human ATG12 ATG5 conjugate required for LC3 lipidation in autophagy. Nat Struct Mol Bio/ 20, 59-66 (2013).

34. Fujioka, Y., Noda, N.N., Nakatogawa, H., Ohsumi, Y. \& Inagaki, F. Dimeric coiled-coil structure of Saccharomyces cerevisiae Atg16 and its functional significance in autophagy. J Biol Chem 285, 1508-15 (2010).

35. Pantoom, S. et al. RAB33B recruits the ATG16L1 complex to the phagophore via a noncanonical RAB binding protein. Autophagy, 1-15 (2020).

36. Gammoh, N., Florey, O., Overholtzer, M. \& Jiang, X.J. Interaction between FIP200 and ATG16L1 distinguishes ULK1 complex-dependent and -independent autophagy. Nature Structural \& Molecular Biology 20, 144-149 (2013).

37. Nishimura, T. et al. FIP200 regulates targeting of Atg16L1 to the isolation membrane. EMBO Rep 14, 284-91 (2013).

38. Fujita, N. et al. Recruitment of the autophagic machinery to endosomes during infection is mediated by ubiquitin. Journal of Cell Biology 203, 115-128 (2013). 
39. Sorbara, M.T. et al. Complement C3 Drives Autophagy-Dependent Restriction of Cyto-invasive Bacteria. Cell Host Microbe 23, 644-652 e5 (2018).

40. King, B.C. et al. Complement Component C3 Is Highly Expressed in Human Pancreatic Islets and Prevents beta Cell Death via ATG16L1 Interaction and Autophagy Regulation. Cell Metabolism 29, 202-+ (2019).

41. Xu, Y. et al. A Bacterial Effector Reveals the V-ATPase-ATG16L1 Axis that Initiates Xenophagy. Cell 178, 552-566 e20 (2019).

42. Gammoh, N. The multifaceted functions of ATG16L1 in autophagy and related processes. J Cell Sci 133(2020).

43. Kelley, L.A., Mezulis, S., Yates, C.M., Wass, M.N. \& Sternberg, M.J. The Phyre2 web portal for protein modeling, prediction and analysis. Nat Protoc 10, 845-58 (2015).

44. Krick, R. et al. Structural and functional characterization of the two phosphoinositide binding sites of PROPPINs, a beta-propeller protein family. Proc Natl Acad Sci U S A 109, E2042-9 (2012).

45. Baskaran, S., Ragusa, M.J., Boura, E. \& Hurley, J.H. Two-site recognition of phosphatidylinositol 3phosphate by PROPPINs in autophagy. Mol Cell 47, 339-48 (2012).

46. Liang, R., Ren, J., Zhang, Y. \& Feng, W. Structural Conservation of the Two PhosphoinositideBinding Sites in WIPI Proteins. J Mol Biol 431, 1494-1505 (2019).

47. Jelani, M. et al. A mutation in the major autophagy gene, WIPI2, associated with global developmental abnormalities. Brain 142, 1242-1254 (2019).

48. Ren, J. et al. Multi-site-mediated entwining of the linear WIR-motif around WIPI beta-propellers for autophagy. Nat Commun 11, 2702 (2020).

49. Hampe, J. et al. A genome-wide association scan of nonsynonymous SNPs identifies a susceptibility variant for Crohn disease in ATG16L1. Nat Genet 39, 207-11 (2007).

50. Murthy, A. et al. A Crohn's disease variant in Atg16/1 enhances its degradation by caspase 3 . Nature 506, 456-+ (2014).

51. Lassen, K.G. et al. Atg16L1 T300A variant decreases selective autophagy resulting in altered cytokine signaling and decreased antibacterial defense. Proc Natl Acad Sci U S A 111, 7741-6 (2014).

52. Wang, Z. et al. Automatic crystal centring procedure at the SSRF macromolecular crystallography beamline. J Synchrotron Radiat 23, 1323-1332 (2016).

53. Otwinowski, Z. \& Minor, W. Processing of X-ray diffraction data collected in oscillation mode. Macromolecular Crystallography, Pt A 276, 307-326 (1997). 
54. Vonrhein, C. et al. Data processing and analysis with the autoPROC toolbox. Acta Crystallogr D Biol Crystallogr 67, 293-302 (2011).

55. Storoni, L.C., McCoy, A.J. \& Read, R.J. Likelihood-enhanced fast rotation functions. Acta Crystallographica Section D-Biological Crystallography 60, 432-438 (2004).

56. Emsley, P. \& Cowtan, K. Coot: model-building tools for molecular graphics. Acta Crystallographica Section D-Biological Crystallography 60, 2126-2132 (2004).

57. Adams, P.D. et al. PHENIX: building new software for automated crystallographic structure determination. Acta Crystallographica Section D-Biological Crystallography 58, 1948-1954 (2002).

58. Davis, I.W. et al. MolProbity: all-atom contacts and structure validation for proteins and nucleic acids. Nucleic Acids Research 35, W375-W383 (2007).

\section{Figures}

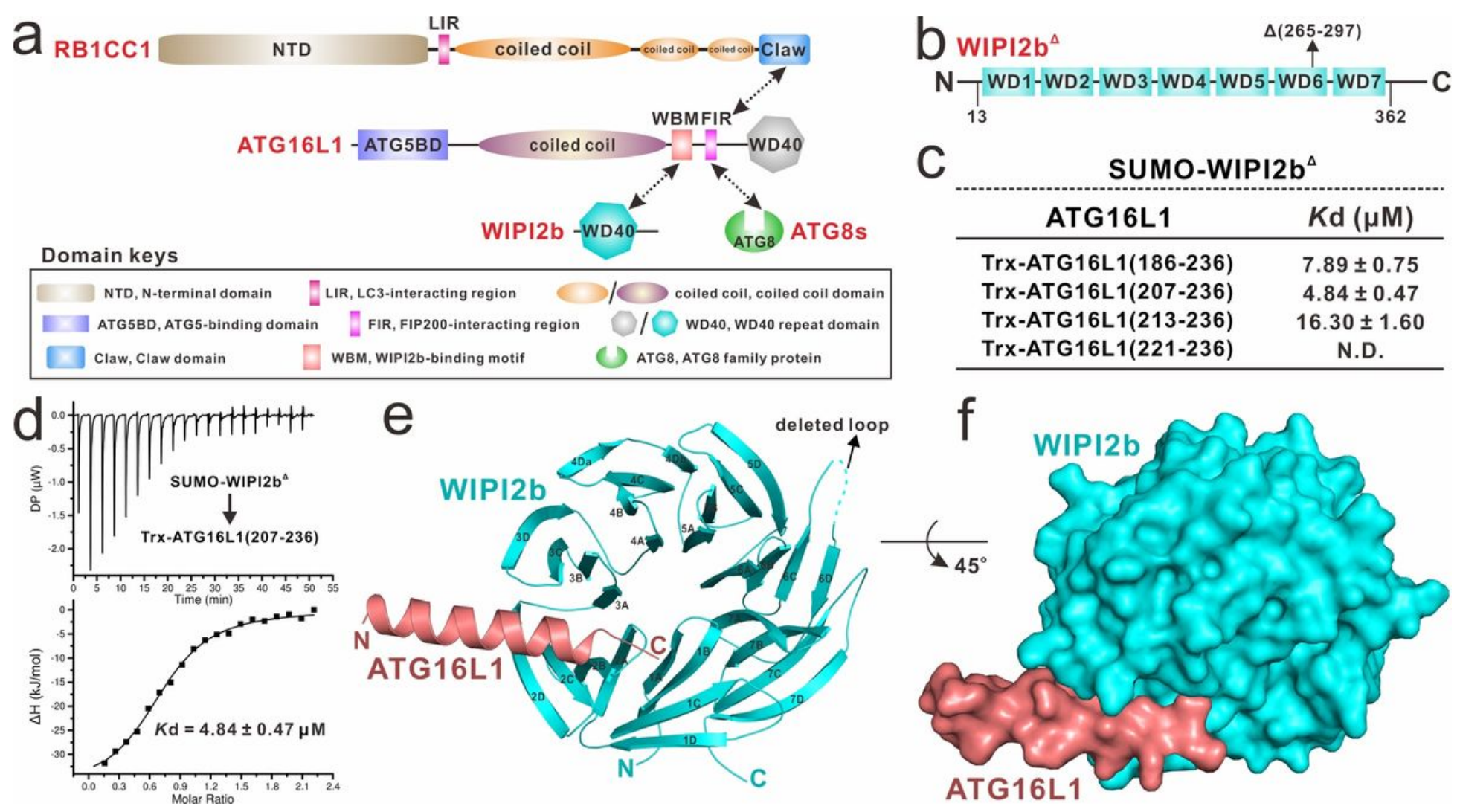

Figure 1

Biochemical and structural characterizations of the interaction between WIPI2b and ATG16L1. (a) A schematic diagram showing the domain organizations of RB1CC1, ATG16L1, WIPI2b and mammalian ATG8 family proteins. In this drawing, the interactions of ATG16L1 with RB1CC1, WIPI2b and mammalian ATG8 family proteins are highlighted and indicated by two-way arrows. (b) A schematic diagram of the 
WIPI2b $\Delta$ (residues 13-362 without 265-297) construct. (c) The measured binding affinities between WIPI2b $\triangle$ and different ATG16L1 fragments by ITC-based binding assays. "N.D." stands for that the Kd value is not detectable. (d) ITC-based measurement of the binding affinity of WIPI2b $\Delta$ with the WIPI2bbinding motif (WBM) of ATG16L1. (e) Ribbon diagram showing the overall structure of the WIPI2b $\Delta$ /ATG16L1 WBM complex. In this drawing, WIPI2b $\Delta$ is shown in cyan and ATG16L1 WBM in deep salmon. The deleted putative membrane-insertion region (residues 265-297) within the sixth blade of WIPI2b WD40 repeats domain is further indicated with a cyan dash line. (f) The surface representation showing the overall architecture of WIPI2b $\triangle$ /ATG16L1WBM complex with the same color scheme as in panel (e). 

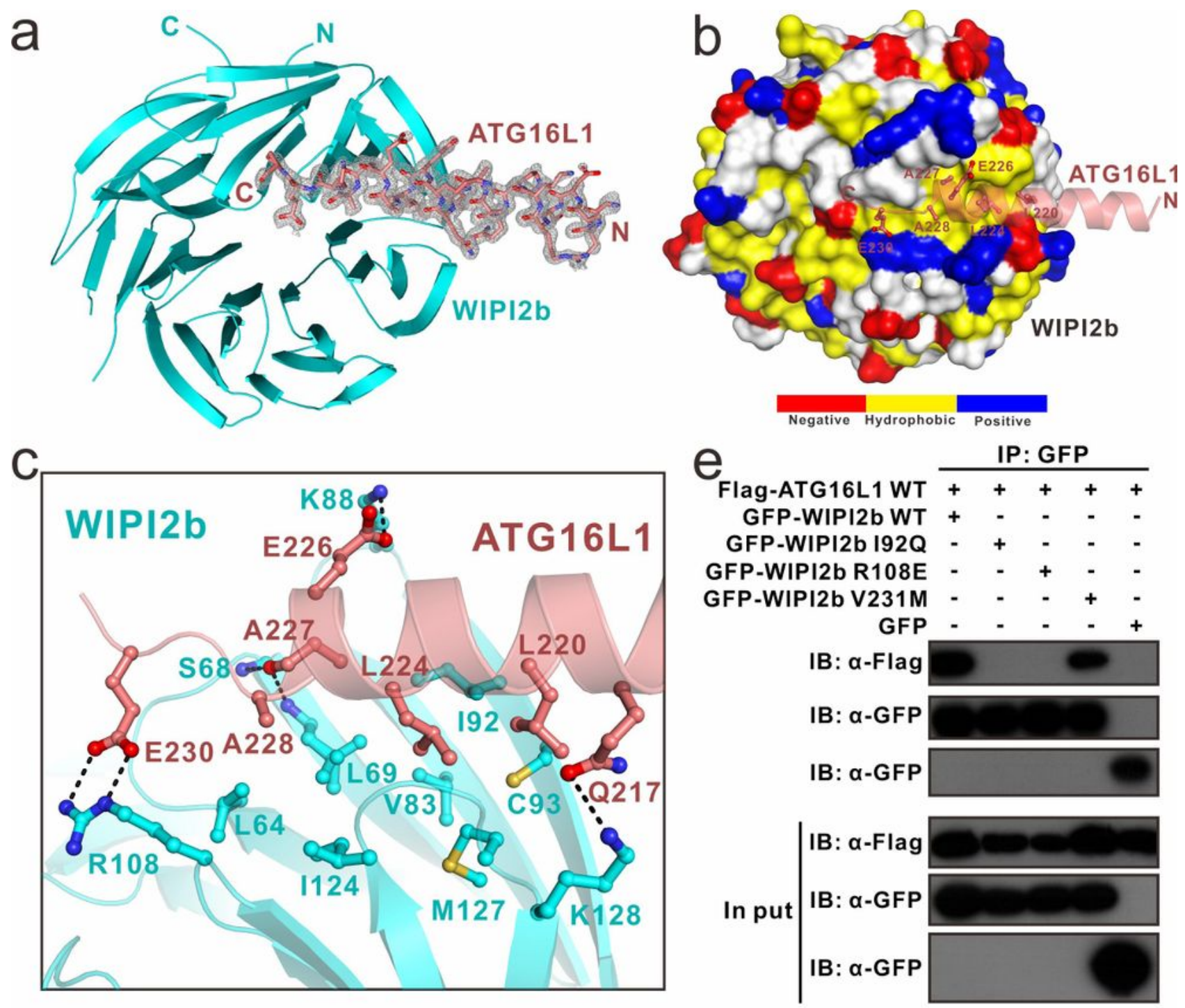

\begin{tabular}{ccc}
\hline d & & \\
ATG16L1 & WIPI2b $^{\Delta}$ & Kd $(\mu M)$ \\
\hline WBR & WT & $6.13 \pm 0.44$ \\
WBR & L69A & N.D. \\
WBR & I92Q & N.D. \\
WBR & R108E & N.D. \\
WBR & V231M & $11.20 \pm 0.65$ \\
WBR(L224Q) & WT & N.D. \\
WBR(A227Q) & WT & N.D. \\
WBR(E226R) & WT & N.D. \\
WBR(E230R) & WT & N.D. \\
\hline
\end{tabular}

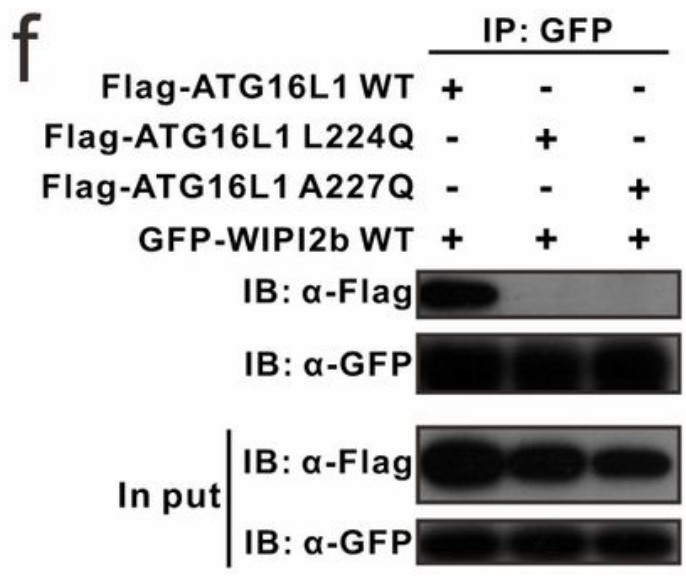

Figure 2

The molecular mechanism of WIPI2b and ATG16L1 interaction. (a) The 2mFo-DFc map of ATG16L1 WBM in the structure of the WIPI2b $\triangle$ /ATG16L1 WBM complex showing that the densities of ATG16L1 WBM can be clearly assigned. The electron density map is calculated by omitting the ATG16L1 WBM peptide from the final PDB file and contoured at 1.6б. (b) The combined surface representation and the ribbon-stick model showing the hydrophobic binding surface between WIPI2b $\triangle$ and ATG16L1 WBM. In 
this drawing, ATG16L1 WBM is displayed as ribbon-stick model, and WIPI2b $\Delta$ is showed as surface representation colored by different amino acid types. Specifically, the hydrophobic amino acid residues in the surface model of WIPI2b $\Delta$ are drawn in yellow, the positively charged residues in blue, the negatively charged residues in red, and the uncharged polar residues in gray. (c) Ribbon-stick model showing the detailed interactions between WIPI2b $\triangle$ and ATG16L1 WBM. The hydrogen bonds and salt bridges involved in the binding are shown as dotted lines. (d) The measured binding affinities between ATG16L1 WBM and WIPI2b $\Delta$, or their mutants by ITC-based binding assays. (e and f) Co-immunoprecipitation assays showing that point mutations of key interface residues of WIPI2b (e) or ATG16L1 (f) observed in the WIPI2b $\triangle$ /ATG16L1 WBM complex structure abolish their specific interaction in cells, while the disease-associated V231M mutation of WIPI2b slightly impairs the binding of WIPI2b with ATG16L1. In these assays, cell extracts were prepared from HEK293T cells co-transfected with different combinations of plasmids as indicated. Here "IB" means immunoblotting.
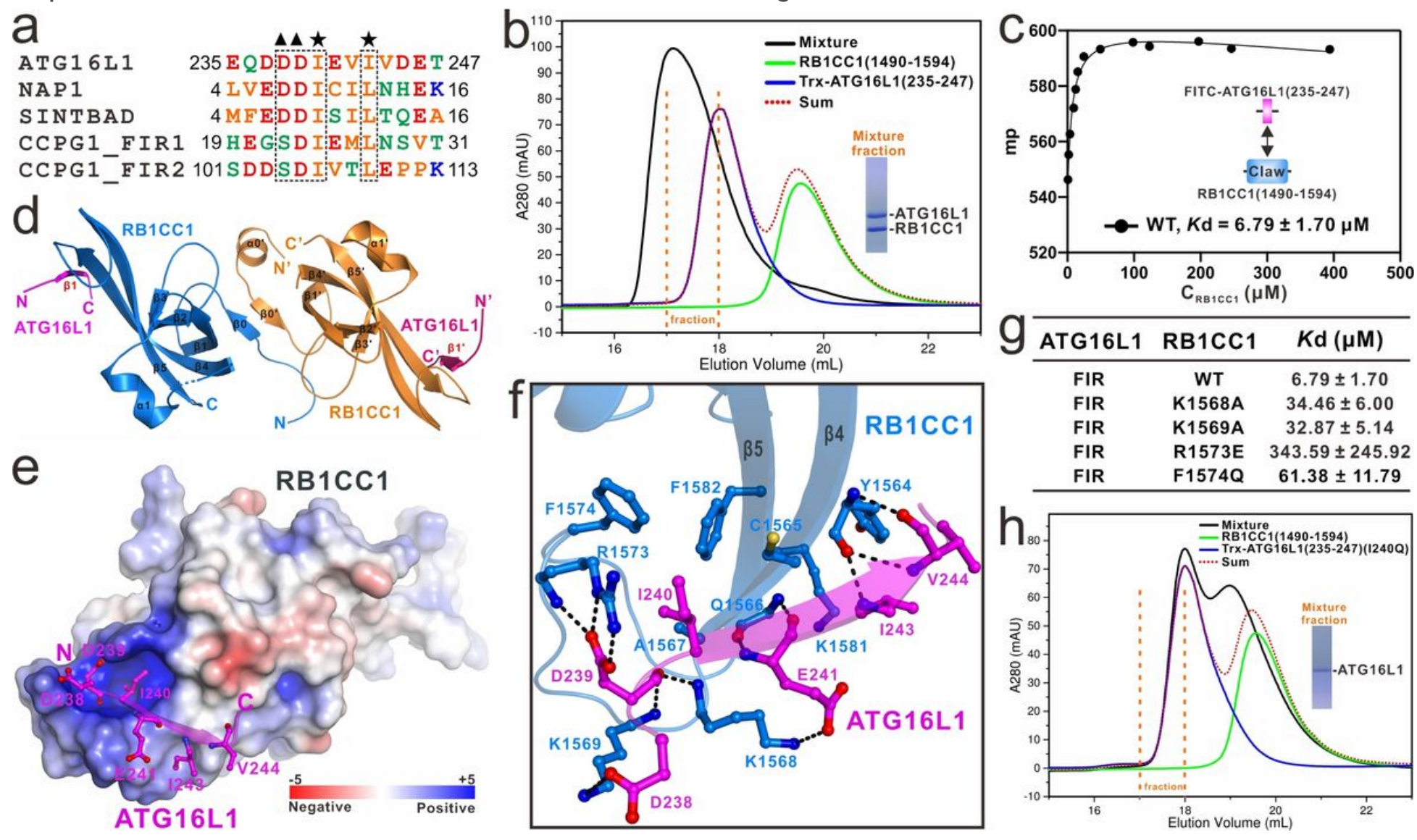

Figure 3

Biochemical and structural characterizations of the interaction between ATG16L1 FIR and RB1CC1 Claw. (a) Sequence alignment analysis of the RB1CC1-interacting-region (FIR) of ATG16L1 with the currently known RB1CC1 Claw-binding regions of NAP1, SINTBAD and CCPG1 from human species. In this alignment, the highly conserved acidic residues (Asp, Glu or potentially phosphorylated Ser residue) and the following two conserved hydrophobic residues are boxed and highlighted with black triangles and stars respectively. (b) Analytic gel filtration chromatography-based analysis of the interaction of RB1CC1 Claw domain with Trx-tagged ATG16L1 FIR. In this panel, "Sum" stands for the theoretical sum of Trx- 
tagged ATG16L1 FIR and RB1CC1 Claw profiles, while "Mixture" stands for the mixture sample of Trxtagged ATG16L1 FIR and RB1CC1 Claw. The insert shows the SDS-PAGE combined with Coomassie blue staining analysis of the protein components of the indicated "Mixture fraction" fraction collected from the analytical gel filtration chromatography experiment of the "Mixture" sample (the black curve). (c) The fluorescence polarization (FP)-based assay measuring the binding affinity of RB1CC1 Claw with FITClabeled ATG16L1 FIR. The Kd value is the fitted dissociation constant with standard errors obtained by using the one-site binding model to fit the FP data. (d) Ribbon diagram showing the overall structure of the dimeric RB1CC1 Claw/ATG16L1 FIR complex. In this drawing, two RB1CC1 Claw molecules are colored in orange and marine, while two bound ATG16L1 FIR motifs are colored in magenta and hot-pink respectively. (e) The combined surface charge representation and the ribbon-stick model showing the charge-charge interactions between RB1CC1 Claw and ATG16L1 FIR in the solved complex structure. (f) Ribbon-stick model showing the detailed interactions between RB1CC1 Claw and ATG16L1 FIR. The hydrogen bonds and salt bridges involved in the interaction are shown as dotted lines. (g) The measured binding affinities between RB1CC1 Claw and ATG16L1 FIR, or their mutants by FP-based binding assays. (h) Analytic gel filtration chromatography-based analysis of the interaction of RB1CC1 Claw domain with the Trx-tagged ATG16L1 FIR I240Q mutant. 


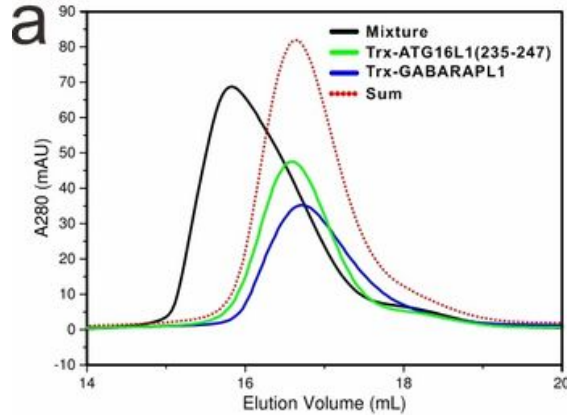

C
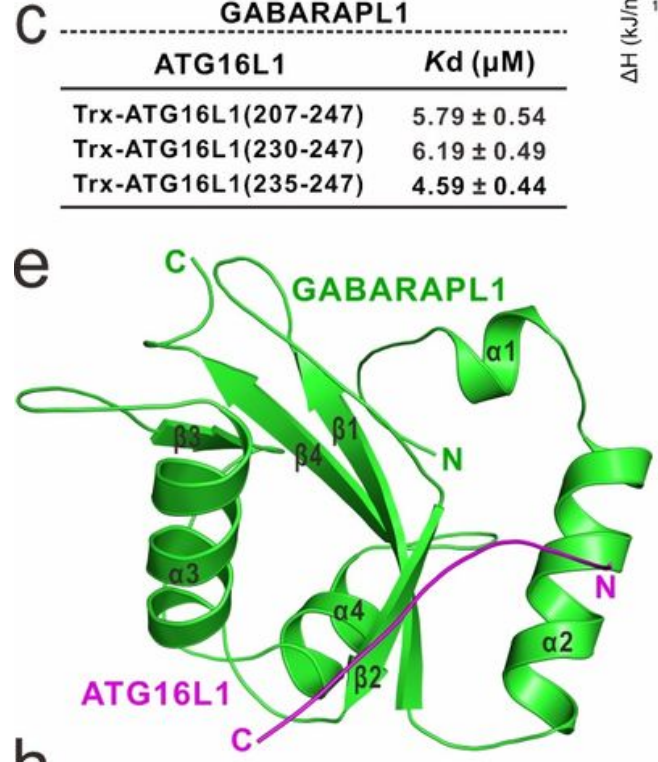

$\mathrm{h}$

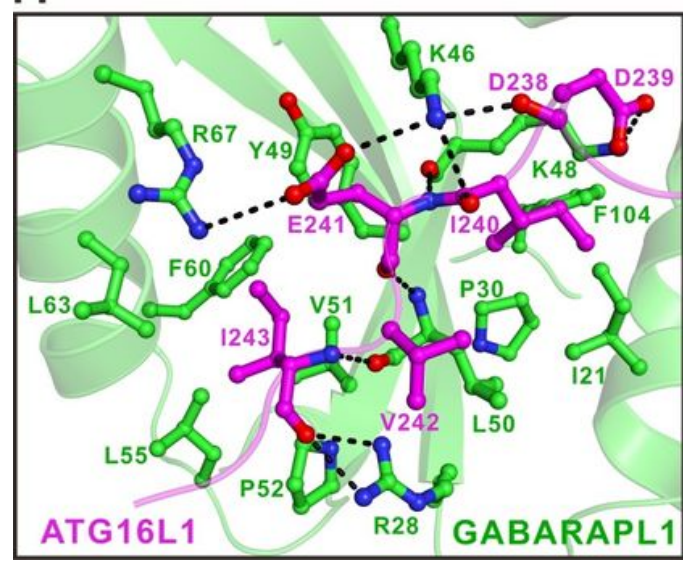

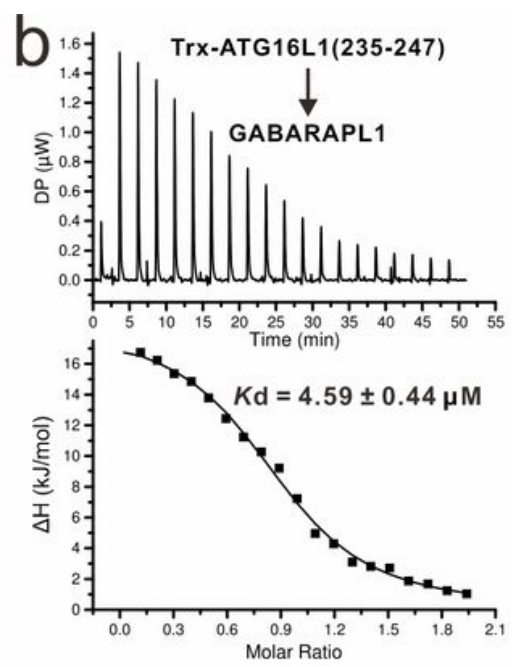
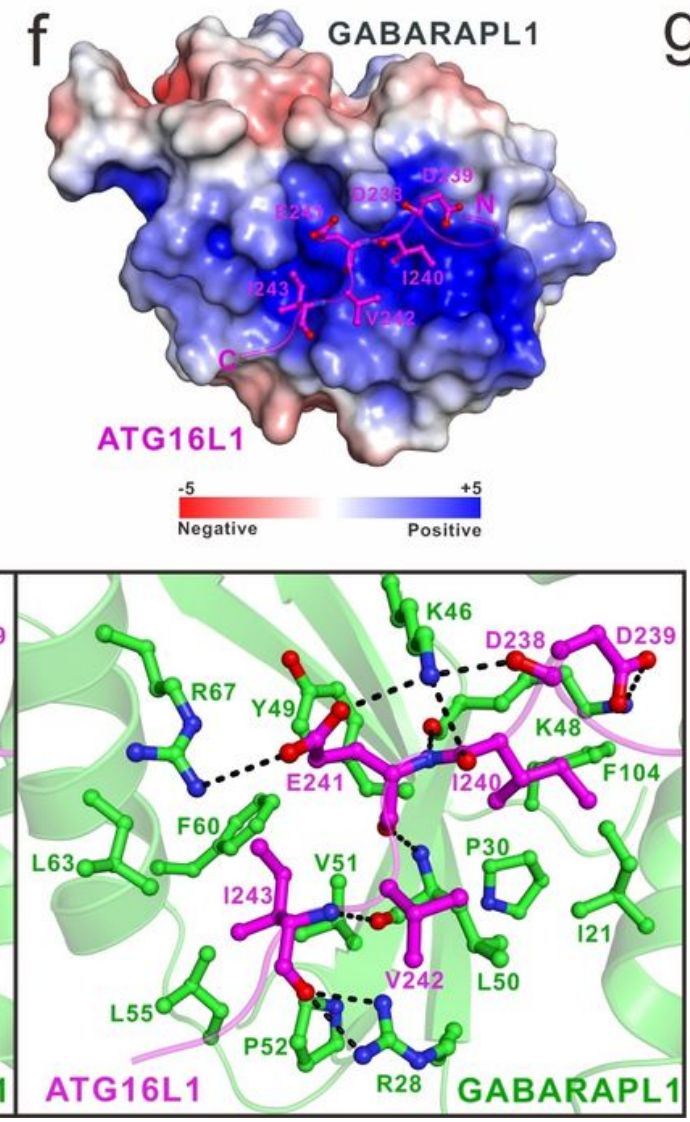
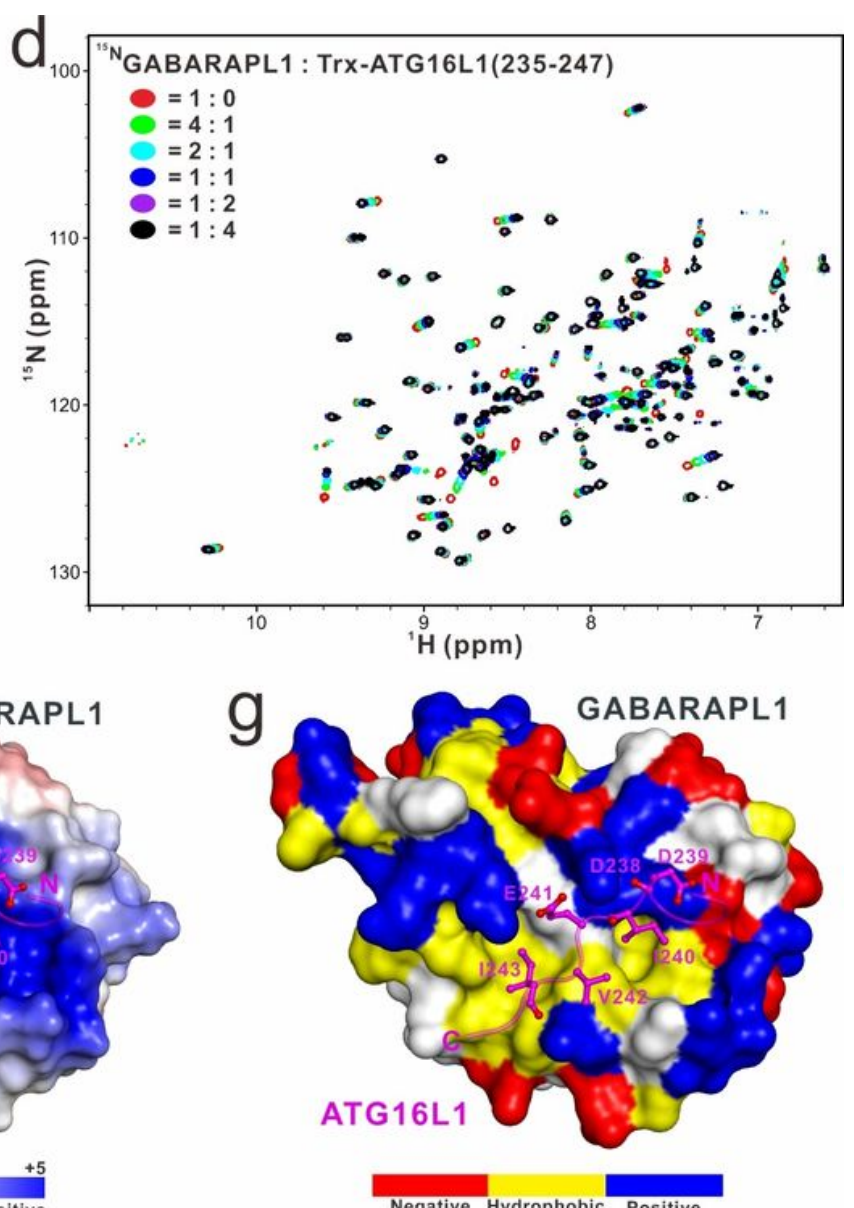

Negative Hydrophobic Positive

\begin{tabular}{ccc}
\hline ATG16L1 & ATG8 & $K \mathrm{~d}(\mu \mathrm{M})$ \\
\hline FIR & LC3A & $7.28 \pm 0.45$ \\
FIR & LC3B & $18.10 \pm 1.61$ \\
FIR & LC3C & $6.27 \pm 0.58$ \\
FIR & GABARAP & $8.11 \pm 0.48$ \\
FIR & GABARAPL1 & $4.59 \pm 0.44$ \\
FIR & GABARAPL2 & $179.00 \pm 41.50$ \\
FIR & GABARAPL1(I21Q) & $11.40 \pm 1.10$ \\
FIR & GABARAPL1(K48E) & $\mathrm{N} . \mathrm{D}$. \\
FIR & GABARAPL1(L50Q) & $41.60 \pm 9.01$ \\
FIR & GABARAPL1(R67E) & $\mathrm{N} . \mathrm{D}$. \\
FIR(D239R) & GABARAPL1 & $76.00 \pm 75.30$ \\
FIR(I240Q) & GABARAPL1 & $85.20 \pm 13.20$ \\
FIR(E241R) & GABARAPL1 & $56.30 \pm 25.90$ \\
FIR(V242E) & GABARAPL1 & $10.50 \pm 0.85$ \\
FIR(I243Q) & GABARAPL1 & $164.00 \pm 140.00$ \\
\hline & &
\end{tabular}

Figure 4

Biochemical and structural characterizations of the interactions of ATG16L1 FIR with mammalian ATG8 family proteins. (a) Analytic gel filtration chromatography-based analysis of the interaction of Trx-tagged GABARAPL1 with Trx-tagged ATG16L1 FIR. (b) ITC-based measurement of the binding affinity of GABARAPL1 with Trx-tagged ATG16L1 FIR. (c) The measured binding affinities of GABARAPL1 with different Trx-tagged ATG16L1 fragments by ITC-based binding assays. (d) Superposition plots of the $1 \mathrm{H}$ 15N HSQC spectra of GABARAPL1 titrated with the un-labeled Trx-tagged ATG16L1 FIR at different molar ratios. (e) Ribbon diagram showing the overall structure of GABARAPL1/ATG16L1 FIR complex. In this drawing, the GABARAPL1 molecule is colored in green, while ATG16L1 FIR in magenta. (f) The combined surface representation and the ribbon-stick model showing the hydrophobic interactions between 
GABARAPL1 and ATG16L1 FIR. In this drawing, ATG16L1 FIR is displayed in the ribbon-stick model, and GABARAPL1 is showed in surface representation colored by amino acid types as shown in Figure 2(b).

(g) The combined surface charge representation and the ribbon-stick model showing the charge-charge interactions between GABARAPL1 and ATG16L1 FIR. (h) Stereo view of the ribbon-stick model showing the detailed interactions between GABARAPL1 and ATG16L1 FIR. The hydrogen bonds and salt bridges involved in the interaction are shown as dotted lines. (i) The measured binding affinities between ATG16L1 FIR and six mammalian ATG8 family proteins or their mutants by ITC-based binding assays.
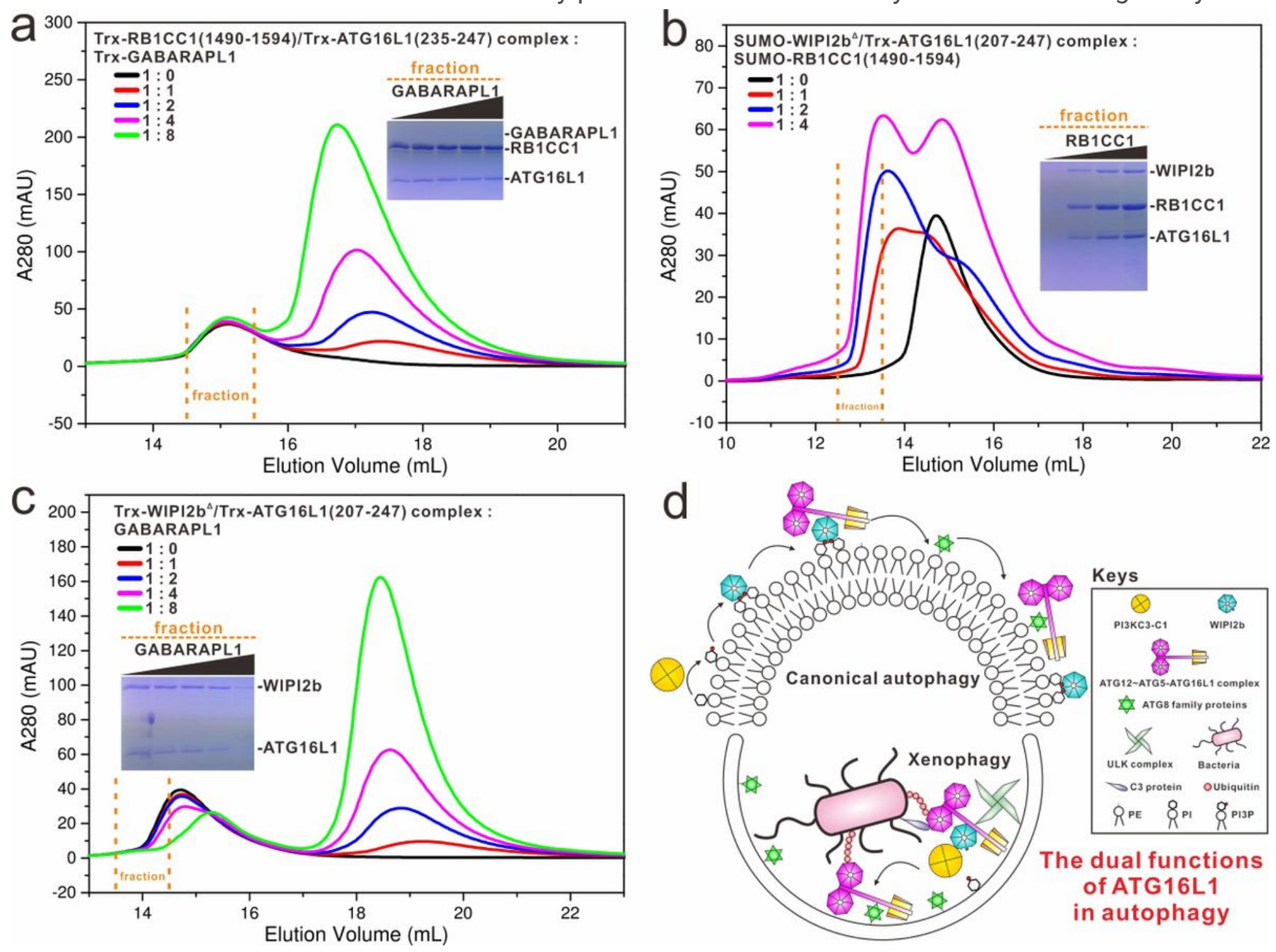

\section{Figure 5}

The relationships of WIPI2b, RB1CC1 and GABARAPL1 in binding to ATG16L1. (a) Analytic gel filtration chromatography coupled with SDS-PAGE analysis of the RB1CC1 Claw/ATG16L1 FIR complex incubated with increasing molar ratio of GABARAPL1 proteins. The insert shows the SDS-PAGE combined with Coomassie blue staining analyses of the protein components of the indicated "fraction" fractions collected from the corresponding analytical gel filtration chromatography experiments with different molar ratios of GABARAPL1. (b) Analytic gel filtration chromatography coupled with SDS-PAGE analysis of the WIPI2b $\Delta$ /ATG16L1(207-247) complex incubated with increasing molar ratio of RB1CC1 Claw 
proteins. The insert shows the SDS-PAGE combined with Coomassie blue staining analyses of the protein components of the indicated "fraction" fractions collected from the corresponding analytical gel filtration chromatography experiments with different molar ratios of RB1CC1 Claw. (c) Analytic gel filtration chromatography coupled with SDS-PAGE analysis of the WIPI2b $\Delta$ /ATG16L1(207-247) complex incubated with increasing molar ratio of GABARAPL1 proteins. The insert shows the SDS-PAGE combined with Coomassie blue staining analyses of the protein components of the indicated "fraction" fractions collected from the corresponding analytical gel filtration chromatography experiment with different molar ratios of GABARAPL1. (d) A proposed model depicting the distinct functions of ATG16L1 in canonical autophagy and xenophagy.

\section{Supplementary Files}

This is a list of supplementary files associated with this preprint. Click to download.

- SupplementalMaterialsNSMBV9.pdf 